\title{
ADDITIVITY OF ELEMENTARY MAPS ON GENERALIZED MATRIX ALGEBRAS
}

\section{DRISS AIAT HADJ AHMED and RACHID TRIBAK}

Centre Régional des Métiers de I'Education

et de la Formation (CRMEF)-Tanger

Avenue My Abdelaziz

Souani, BP: 3117, Tanger

Morocco

e-mail: ait_hadj@yahoo.com

\begin{abstract}
Let $\mathcal{R}$ be a commutative ring with identity, $\mathcal{A}$ and $\mathcal{B}$ be two algebras (maybe without identity) over $\mathcal{R}$. Let $\mathcal{M}$ be an $(\mathcal{A}, \mathcal{B})$-bimodule, $\mathcal{N}$ be a $(\mathcal{B}, \mathcal{A})$-bimodule, and $\mathcal{G}$ be the generalized matrix algebra. In this paper, it is proved that under some conditions, every $\left(M, M^{*}\right)$ is an elementary map on $\mathcal{G} \times \mathcal{R}^{\prime}$, and both $M$ and $M^{*}$ are surjective. Then both $M$ and $M^{*}$ are additive.
\end{abstract}

\section{Introduction}

Let $\mathcal{R}$ be a commutative ring with identity, $\mathcal{A}$ and $\mathcal{B}$ be two algebras (maybe without identity) over $\mathcal{R}$. Let $\mathcal{M}$ be an $(\mathcal{A}, \mathcal{B})$ bimodule and $\mathcal{N}$ be a $(\mathcal{B}, \mathcal{A})$-bimodule. Assume that there are two bimodule homomorphisms $\varphi: \mathcal{M} \otimes_{B} \mathcal{N} \rightarrow \mathcal{A}$ and $\psi: \mathcal{N} \otimes_{A} \mathcal{M} \rightarrow \mathcal{B}$ 
satisfying the following associativity conditions: $(m n) m^{\prime}=m\left(n m^{\prime}\right)$ and $(n m) n^{\prime}=n\left(m n^{\prime}\right)$ for all $m, m^{\prime} \in \mathcal{M}$ and $n, n^{\prime} \in \mathcal{N}$, where we put $m n=$ $\varphi(m \otimes n)$ and $n m=\psi(n \otimes m)$.

A generalized matrix algebra $\mathcal{G}=\operatorname{Mat}(\mathcal{A}, \mathcal{M}, \mathcal{N}, \mathcal{B})$ is an algebra of the form

$$
\begin{aligned}
\operatorname{Mat}(\mathcal{A}, \mathcal{M}, \mathcal{N}, \mathcal{B})=\mathcal{G} & =\left(\begin{array}{ll}
\mathcal{A} & \mathcal{M} \\
\mathcal{N} & \mathcal{B}
\end{array}\right) \\
& =\left\{\left(\begin{array}{cc}
a & m \\
n & b
\end{array}\right): a \in \mathcal{A}, m \in \mathcal{M}, b \in \mathcal{B}, n \in \mathcal{N}\right\},
\end{aligned}
$$

under the usual matrix-like addition and matrix-like multiplication, where at least one of the two bimodules $\mathcal{M}$ and $\mathcal{N}$ is distinct from zero. Obviously, the triangular algebras studied in [1] are a kind of generalized matrix algebras. We refer the reader to [6, Section 2] for some classical examples of generalized matrix algebras.

Let $\mathcal{G}_{i j}=e_{i} \mathcal{G} e_{j}$ for all $1 \leq i, j \leq 2$. It is clear that $\mathcal{G}$ can be represented as $\mathcal{G}=\mathcal{G}_{11} \oplus \mathcal{G}_{12} \oplus \mathcal{G}_{21} \oplus \mathcal{G}_{22}$. Moreover, $\mathcal{G}_{11} \simeq \mathcal{A}, \mathcal{G}_{12} \simeq \mathcal{M}$, $\mathcal{G}_{21} \simeq \mathcal{N}$, and $\mathcal{G}_{22} \simeq \mathcal{B}$.

Recall that a left $\mathcal{A}$-module (resp., right $\mathcal{B}$-module) $\mathcal{M}$ is faithful if for any $a \in \mathcal{A}, a \mathcal{M}=\{0\}$ (resp., for any $b \in \mathcal{B}, b \mathcal{M}=\{0\}$ ) implies $a=0$ (resp., $b=0)$.

Definition ([2]). Let $R$ and $R^{\prime}$ be rings. The ordered pair $\left(M, M^{*}\right)$ of maps $M: R \rightarrow R^{\prime}$ and $M^{*}: R^{\prime} \rightarrow R$ is called an elementary map of $R \times R^{\prime}$, if

$$
M\left(a M^{*}(x) b\right)=M(a) x M(b) \text { and } M^{*}(x M(a) y)=M^{*}(x) a M^{*}(y),
$$

for all $a, b \in R, x, y \in R^{\prime}$. 
Elementary maps were originally introduced by Bresar and Serml in their nice paper [2]. There are many examples of elementary maps. Obviously, if $\Phi$ is a bijective map from $R$ into $R^{\prime}$ such that $\Phi(x \circ y)=$ $\Phi(x) \circ \Phi(y)$ for any $x, y \in R$, then the ordered pair $\left(\Phi, \Phi^{-1}\right)$ is a Jordan map of $R \times R^{\prime}$. For $a, b \in R$, let $M_{a, b}(x)=a x b$ for $x \in R$. Then one can verify that $\left(M_{a, b}, M_{b, a}\right)$ is an elementary map on $R \times R$. The additivity of elementary maps on operator algebras were studied in [2], [1], [3], and $[4]$.

\section{The Results}

The following is our main result in this section. The aim of this section is to study the additivity of elementary maps on generalized matrix algebras. We now state our first main result.

Theorem 2.1. Let $\mathcal{R}$ be a commutative ring with identity, $\mathcal{A}$ and $\mathcal{B}$ be two algebras (maybe without identity) over $\mathcal{R}$. Let $\mathcal{M}$ be an $(\mathcal{A}, \mathcal{B})$ bimodule, $\mathcal{N}$ be a $(\mathcal{B}, \mathcal{A})$-bimodule, and $\mathcal{G}$ be the generalized matrix algebra. Suppose that $\mathcal{A}, \mathcal{B}$, and $\mathcal{M}$ satisfy:

(i) For $a \in \mathcal{A}$, if $a \mathcal{A}=\{0\}$, or $\mathcal{A} a=\{0\}$, then $a=0$.

(ii) For $b \in \mathcal{B}$, if $b \mathcal{B}=\{0\}$, or $\mathcal{B} b=\{0\}$, then $b=0$.

(iii) For $m \in M$, if $\mathcal{A} m=\{0\}$, or $m \mathcal{B}=\{0\}$, then $m=0$.

Suppose that $\left(M, M^{*}\right)$ is an elementary map on $\mathcal{G} \times \mathcal{R}^{\prime}$, and both $M$ and $M^{*}$ are surjective. Then both $M$ and $M^{*}$ are additive.

Lemma 2.2. $M(0)=0$ and $M^{*}(0)=0$.

Proof. $M(0)=M\left(0 M^{*}(0) 0\right)=M(0) 0 M(0)=0$. Similarly, we can show that $M^{*}(0)=0$. 
Lemma 2.3. If $\mathcal{G} u \mathcal{G}=0$ for some $u \in \mathcal{G}$, then $u=0$.

Lemma 2.4. Both $M$ and $M^{*}$ are bijective.

Proof. It suffices to prove that $M$ and $M^{*}$ are injective. First establish the injectivity of $M$.

Suppose that $M(a)=M(b)$ for some $a, b \in \mathcal{G}$. By assumption, we have

$$
M^{*}(x) a M^{*}(y)=M^{*}(x M(a) y)=M^{*}(x M(b) y)=M^{*}(x) b M^{*}(y) .
$$

Thus $M^{*}(x)(a-b) M^{*}(y)=0$ for all $x, y \in R^{\prime}$. Since $M^{*}$ is surjective, we get that $\mathcal{G}(a-b) \mathcal{G}=0$ and so $a-b=0$ by Lemma 2.3. Thus $a=b$. Then $M$ is injective.

$$
M\left(a M^{*}(x) b\right)=M(a) x M(b) \text { and } M^{*}(x M(a) y)=M^{*}(x) a M^{*}(y) .
$$

Now we turn to proving the injectivity of $M^{*}$. Suppose that $M^{*}(x)=M^{*}(y)$ for some $x, y \in R^{\prime}$. Since $M$ is surjective, we get that there exist $a, b \in \mathcal{G}$ such that $M(a)=x$ and $M(b)=y$. For every $z \in \mathcal{G}$, we have

Now for any $a, b \in \mathcal{G}$, we have

$$
\begin{aligned}
M^{*} M(a) M^{-1}(x) M^{*} M(b) & =M^{*}\left(M(a) M M^{-1}(x) M(b)\right) \\
& =M^{*}(M(a) x M(b)) \\
& =M^{*} M\left(a M^{*}(x) b\right) \\
& =M^{*} M\left(a M^{*}(y) b\right) \\
& =M^{*}(M(a) y M(b)) \\
& =M^{*}\left(M(a) M M^{-1}(y) M(b)\right) .
\end{aligned}
$$

Thus $M^{*} M(a) M^{-1}(x) M^{*} M(b)=M^{*} M(a) M^{-1}(y) M^{*} M(b)$. 
Equivalently, since $M^{*} M$ is surjective. $\mathcal{G}\left(M^{-1}(x)-M^{-1}(y)\right) \mathcal{G}=0$.

It follows from the same argument above that $M^{-1}(x)=M^{-1}(y)$, and so $x=y$, as desired.

Lemma 2.5. The pair $\left(M^{*-1}, M^{-1}\right)$ is an elementary map on $\mathcal{G} \times \mathcal{R}^{\prime}$. That is,

$$
\begin{aligned}
& M^{-1}\left(x M^{*-1}(a) y\right)=M^{-1}(x) a M^{-1}(y), \\
& M^{*-1}\left(a M^{-1}(x) b\right)=M^{*-1}(a) x M^{*-1}(b) .
\end{aligned}
$$

Lemma 2.6. Let $a, b, c \in \mathcal{G}$.

(i) If $M(c)=M(a)+M(b)$, then

$$
M^{*-1}(s c t)=M^{*-1}(s a t)+M^{*-1}(s b t) \text { for all } s, t \in \mathcal{G}
$$

(ii) If $M^{*-1}(c)=M^{*-1}(a)+M^{*-1}(b)$, then

$$
M(s c t)=M(s a t)+M(s b t) \text { for all } s, t \in \mathcal{G} .
$$

Proof. We only prove (i), and (ii) goes similarly.

By Lemma 2.4, we have

$$
\begin{aligned}
M^{*-1}(s a t) & =M^{*-1}\left(s M^{-1} M(c) t\right)=M^{*-1}(s) M(c) M^{*-1}(t) \\
& =M^{*-1}(s)(M(a)+M(b)) M^{*-1}(t) \\
& =\left(M^{*-1}(s) M(a) M^{*-1}(t)\right)+\left(M^{*-1}(s) M(b) M^{*-1}(t)\right) \\
& =M^{*-1}(s a t)+M^{*-1}(s b t) .
\end{aligned}
$$

Lemma 2.7. $M\left(a_{11}+a_{12}+a_{21}+a_{22}\right)=M\left(a_{11}\right)+M\left(a_{12}\right)+M\left(a_{21}\right)$ $+M\left(a_{22}\right)$ for every $A_{i j} \in \mathcal{G}_{i j}$. 
Proof. Let $c \in \mathcal{G}$ be an element such that $M(C)=M\left(a_{11}\right)+M\left(a_{12}\right)$ $+M\left(a_{21}\right)+M\left(a_{22}\right)$.

$$
M^{*-1}(s c t)=M^{*-1}\left(s a_{11} t\right)+M^{*-1}\left(s a_{12} t\right)+M^{*-1}\left(s a_{21} t\right)+M^{*-1}\left(s a_{22} t\right),
$$

for all $s, t \in \mathcal{G}$.

Then we have for every $x_{i j}, 1 \leq i, j \leq 2$

$$
\begin{aligned}
M^{*-1}\left(x_{i j} c x_{i j}\right) & =\sum_{l, t} M^{*-1}\left(x_{i j} a_{l t} x_{i j}\right) \\
& =M^{*-1}\left(x_{i j} a_{j i} x_{i j}\right) .
\end{aligned}
$$

Hence it follows that $x_{i j} c x_{i j}=x_{i j} a_{j i} x_{i j}$, that is, $x_{i j}\left(c-a_{j i}\right) x_{i j}=0$.

From $x_{i i}\left(c-a_{i i}\right) x_{i i}=0$, since $A$ and $B$ are unital algebras we have $c_{i i}=a_{i i}$ for $1 \leq i \leq 2$.

From $x_{12}\left(c-a_{21}\right) x_{12}=0, c_{21}=a_{21}$ by condition (2). Similarly, we have $c_{12}=a_{12}$.

Lemma 2.8. $M^{*-1}\left(a_{11}+a_{12}+a_{21}+a_{22}\right)=M^{*-1}\left(a_{11}\right)+M^{*-1}\left(a_{12}\right)$ $+M^{*-1}\left(a_{21}\right)+M^{*-1}\left(a_{22}\right)$ for every $a_{i j} \in \mathcal{G}_{i j}$.

Lemma 2.9. For any $t_{11}, a_{11} \in \mathcal{G}_{11}, b_{12}, c_{12} \in \mathcal{G}_{12}$, and $d_{22} \in \mathcal{G}_{22}$, we have

(i) $M\left(t_{11} a_{11} b_{12}+t_{11} c_{12} d_{22}\right)=M\left(t_{11} a_{11} b_{12}\right)+M\left(t_{11} c_{12} d_{22}\right)$,

(ii) $M^{*-1}\left(t_{11} c_{12} d_{22}+t_{11} c_{12} d_{22}\right)=M^{*-1}\left(t_{11} c_{12} d_{22}\right)+M^{*-1}\left(t_{11} c_{12} d_{22}\right)$.

Proof. We only prove (i). Using Lemmas 2.6 and 2.7, we compute

$$
\begin{aligned}
M\left(t_{11} a_{11} b_{12}+t_{11} c_{11} d_{22}\right) & =M\left(t_{11}\left(a_{11}+c_{12}\right)\left(b_{12}+d_{22}\right)\right) \\
& =M\left(t_{11} M^{*} M^{*-1}\left(a_{11}+c_{12}\right)\left(b_{12}+d_{22}\right)\right) \\
& =M\left(t_{11}\right) M^{*-1}\left(a_{11}+c_{12}\right) M\left(b_{12}+d_{22}\right)
\end{aligned}
$$




$$
\begin{aligned}
= & M\left(t_{11}\right)\left[M^{*-1}\left(a_{11}\right)\right. \\
& \left.+M^{*-1}\left(c_{12}\right)\right]\left[M\left(b_{12}\right)+M\left(d_{22}\right)\right] \\
= & M\left(t_{11}\right) M^{*-1}\left(a_{11}\right) M\left(b_{12}\right) \\
& +M\left(t_{11}\right) M^{*-1}\left(a_{11}\right) M\left(d_{22}\right) \\
& +M\left(t_{11}\right) M^{*-1}\left(c_{12}\right) M\left(b_{12}\right) \\
& +M\left(t_{11}\right) M^{*-1}\left(c_{12}\right) M\left(d_{22}\right) \\
= & M\left(t_{11}\right)\left[M^{*-1}\left(a_{11}\right)+M^{*-1}\left(c_{12}\right)\right] M\left(b_{12}\right) \\
& +M\left(t_{11}\right)\left[M^{*-1}\left(a_{11}\right)+M^{*-1}\left(c_{12}\right)\right] M\left(d_{22}\right) \\
= & M\left(t_{11}\right) M^{*-1}\left(a_{11}+c_{12}\right) M\left(b_{12}\right) \\
& +M\left(t_{11}\right) M^{*-1}\left(a_{11}+c_{12}\right) M\left(d_{22}\right) \\
= & M\left(t_{11}\left(a_{11}+c_{12}\right) b_{12}\right)+M\left(\left(t_{11}\left(a_{11}+c_{12}\right) d_{22}\right)\right) \\
= & M\left(t_{11} a_{11} b_{12}\right)+M\left(t_{11} c_{12} d_{22}\right) .
\end{aligned}
$$

Lemma 2.10. $M$ is additive on $\mathcal{G}_{12}$.

Proof. Let $a_{12}$ and $b_{12}$ be in $\mathcal{G}_{12}$. We pick $\mathcal{C} \in \mathcal{G}_{12}$ such that $M(c)=M\left(a_{12}\right)+M\left(b_{12}\right)$.

$$
M^{*-1}(s c t)=M^{*-1}\left(s a_{12} t\right)+M^{*-1}\left(s b_{12} t\right) \text { for all } s, t \in \mathcal{G} \text {. }
$$

Then we have for every $x_{i j}, y_{k l}$ on $\mathcal{G}, 1 \leq i, j, k, l \leq 2$

$$
M^{*-1}\left(x_{i j} c y_{k l}\right)=M^{*-1}\left(x_{i j} a_{12} y_{k l}\right)+M^{*-1}\left(x_{i j} b_{12} y_{k l}\right)
$$

for $(i, j)=(1,1)$ and $(k, l)=(1,1)$ we have $M^{*-1}\left(x_{11} c y_{11}\right)=0$, this implies that $x_{11} c y_{11}=0$, and so $c_{11}=0$.

Similarly, we can get $c_{22}=0$. 
For $(i, j)=(2,2)$ and $(k, l)=(1,1)$ we have $M^{*-1}\left(x_{22} c y_{11}\right)=0$, this implies that $x_{22} c y_{11}=0$, and so $c_{21}=0$.

We now show that $c_{12}=a_{12}+b_{12}$. For any $t_{11}, r_{11} \in \mathcal{G}_{11}$ and $s_{22} \in \mathcal{G}_{22}$, we obtain

$$
\begin{aligned}
M^{*-1}\left(r_{11} t_{11} c s_{22}\right) & =M^{*-1}\left(r_{11} t_{11} a_{12} s_{22}\right)+M^{*-1}\left(r_{11} t_{11} b_{12} s_{22}\right) \\
& =M^{*-1}\left(r_{11} t_{11} a_{12} s_{22}+r_{11} t_{11} b_{12} s_{22}\right) \\
& =M^{*-1}\left(r_{11} t_{11}\left(a_{12}+b_{12}\right) s_{22}\right) .
\end{aligned}
$$

It follows that $r_{11} t_{11} c s_{22}=r_{11} t_{11}\left(a_{12}+b_{12}\right) s_{22}$.

Then we get $c_{12}=a_{12}+b_{12}$.

Lemma 2.11. For any $t_{22}, a_{22} \in \mathcal{G}_{22}, b_{21}, c_{21} \in \mathcal{G}_{21}$, and $d_{11} \in \mathcal{G}_{11}$, we have

(i) $M\left(t_{22} a_{22} b_{21}+t_{22} c_{21} d_{11}\right)=M\left(t_{22} a_{22} b_{21}\right)+M\left(t_{22} c_{21} d_{11}\right)$,

(ii) $M^{*-1}\left(t_{22} a_{22} b_{21}+t_{22} c_{21} d_{11}\right)=M^{*-1}\left(t_{22} a_{22} b_{21}\right)+M^{*-1}\left(t_{22} c_{21} d_{11}\right)$.

Proof. We only prove (i). Using Lemmas 2.6 and 2.7, we compute

$$
\begin{aligned}
M\left(t_{22} a_{22} b_{21}+t_{22} c_{21} d_{11}\right)= & M\left(t_{22}\left(a_{22}+c_{21}\right)\left(b_{21}+d_{11}\right)\right) \\
= & M\left(t_{22} M^{*} M^{*-1}\left(a_{22}+c_{21}\right)\left(b_{21}+d_{11}\right)\right) \\
= & M\left(t_{22}\right) M^{*-1}\left(a_{22}+c_{21}\right) M\left(b_{21}+d_{11}\right) \\
= & M\left(t_{22}\right)\left[M^{*-1}\left(a_{22}\right)\right. \\
& \left.+M^{*-1}\left(a_{21}\right)\right]\left[M\left(b_{21}\right)+M\left(d_{11}\right)\right] \\
= & M\left(t_{22}\right) M^{*-1}\left(a_{22}\right) M\left(b_{21}\right) \\
& +M\left(t_{22}\right) M^{*-1}\left(a_{22}\right) M\left(d_{11}\right) \\
& +M\left(t_{22}\right) M^{*-1}\left(c_{21}\right) M\left(b_{21}\right)
\end{aligned}
$$




$$
\begin{aligned}
& +M\left(t_{22}\right) M^{*-1}\left(c_{21}\right) M\left(d_{11}\right) \\
= & M\left(t_{22}\right)\left[M^{*-1}\left(a_{22}\right)+M^{*-1}\left(c_{21}\right)\right] M\left(b_{21}\right) \\
& +M\left(t_{22}\right)\left[M^{*-1}\left(a_{22}\right)+M^{*-1}\left(c_{21}\right)\right] M\left(d_{11}\right) \\
= & M\left(t_{22}\right) M^{*-1}\left(a_{22}+c_{21}\right) M\left(b_{21}\right) \\
& +M\left(t_{22}\right) M^{*-1}\left(a_{22}+c_{21}\right) M\left(d_{11}\right) \\
= & M\left(t_{22}\left(a_{22}+c_{21}\right) b_{21}\right)+M\left(t_{22}\left(a_{22}+c_{21}\right) d_{11}\right) \\
= & M\left(t_{22} a_{22} b_{21}\right)+M\left(t_{22} c_{21} d_{11}\right) .
\end{aligned}
$$

Lemma 2.12. $M$ is additive on $\mathcal{G}_{21}$.

Proof. Let $a_{21}$ and $b_{21}$ be in $\mathcal{G}_{21}$. We pick $\mathcal{C} \in \mathcal{G}_{21}$ such that $M(c)=M\left(a_{21}\right)+M\left(b_{21}\right)$.

$$
M^{*-1}(s c t)=M^{*-1}\left(s a_{21} t\right)+M^{*-1}\left(s b_{21} t\right) \text { for all } s, t \in \mathcal{G} \text {. }
$$

Then we have for every $x_{i j}, y_{k l}$ on $\mathcal{G}, 1 \leq i, j, k, l \leq 2$

$$
M^{*-1}\left(x_{i j} c y_{k l}\right)=M^{*-1}\left(x_{i j} a_{21} y_{k l}\right)+M^{*-1}\left(x_{i j} b_{21} y_{k l}\right)
$$

for $(i, j)=(2,2)$ and $(k, l)=(2,2)$ we have $M^{*-1}\left(x_{22} c y_{22}\right)=0$, this implies that $x_{22} c y_{22}=0$, and so $c_{22}=0$.

Similarly, we can get $c_{11}=0$.

For $(i, j)=(1,1)$ and $(k, l)=(2,2)$ we have $M^{*-1}\left(x_{11} c y_{22}\right)=0$, this implies that $x_{11} c y_{22}=0$, and so $c_{12}=0$.

We now show that $c_{21}=a_{21}+b_{21}$. For any $t_{22}, r_{22} \in \mathcal{G}_{22}$ and $s_{11} \in \mathcal{G}_{11}$, we obtain

$$
M^{*-1}\left(r_{22} t_{22} c s_{11}\right)=M^{*-1}\left(r_{22} t_{22} a_{21} s_{11}\right)+M^{*-1}\left(r_{22} t_{22} b_{21} s_{11}\right)
$$




$$
\begin{aligned}
& =M^{*-1}\left(r_{22} t_{22} a_{21} s_{11}+r_{22} t_{22} b_{21} s_{11}\right) \\
& =M^{*-1}\left(r_{22} t_{22}\left(a_{21}+b_{21}\right) s_{11}\right) .
\end{aligned}
$$

It follows that

$$
r_{22} t_{22} c s_{11}=r_{22} t_{22}\left(a_{21}+b_{21}\right) s_{11} \text {. }
$$

Then we get $c_{21}=a_{21}+b_{21}$.

Lemma 2.13. $M$ is additive on $\mathcal{G}_{11}$.

Proof. Let $a_{11}$ and $b_{11}$ be in $\mathcal{G}_{11}$. We pick $\mathcal{C} \in \mathcal{G}$ such that $M(c)=M\left(a_{11}\right)+M\left(b_{11}\right)$.

For any $t_{11} \in \mathcal{G}_{11}$ and $s_{12} \in \mathcal{G}_{12}$, we obtain

$$
\begin{aligned}
M^{*-1}\left(t_{11} c s_{12}\right) & =M^{*-1}\left(t_{11} a_{11} s_{12}\right)+M^{*-1}\left(t_{11} b_{11} s_{12}\right) \\
& =M^{*-1}\left(t_{11} a_{11} s_{12}+t_{11} b_{11} s_{12}\right) \\
& =M^{*-1}\left(t_{11}\left(a_{11}+b_{11}\right) s_{12}\right) .
\end{aligned}
$$

It follows that $t_{11} c s_{12}=t_{11}\left(a_{11}+b_{11}\right) s_{12}$.

Then we get $c_{11}=a_{11}+b_{11}$.

Lemma 2.14. $M$ is additive on $\mathcal{G}_{22}$.

Proof. Let $a_{22}$ and $b_{22}$ be in $\mathcal{G}_{22}$. We pick $\mathcal{C} \in \mathcal{G}$ such that $M(c)=M\left(a_{22}\right)+M\left(b_{22}\right)$.

For any $t_{12} \in \mathcal{G}_{12}$ and $s_{22} \in \mathcal{G}_{22}$, we obtain

$$
\begin{aligned}
M^{*-1}\left(t_{12} c s_{22}\right) & =M^{*-1}\left(t_{12} a_{22} s_{22}\right)+M^{*-1}\left(t_{12} b_{22} s_{22}\right) \\
& =M^{*-1}\left(t_{12} a_{22} s_{22}+t_{12} b_{22} s_{22}\right) \\
& =M^{*-1}\left(t_{12}\left(a_{22}+b_{22}\right) s_{22}\right)
\end{aligned}
$$

It follows that $t_{12} c s_{22}=t_{12}\left(a_{22}+b_{22}\right) s_{22}$. 
Then we get $c_{22}=a_{22}+b_{22}$.

Proof of Theorem. We first show the additivity of $M$. Let $a=a_{11}+$ $a_{12}+a_{21}+a_{22}$ and $b=b_{11}+b_{12}+b_{21}+b_{22}$ be two arbitrary elements of $\mathcal{G}$. We have

$$
\begin{aligned}
M(a+b)= & M\left(\left(a_{11}+b_{11}\right)+\left(a_{12}+b_{12}\right)+\left(a_{21}+b_{21}\right)+\left(a_{22}+b_{22}\right)\right) \\
= & M\left(a_{11}+b_{11}\right)+M\left(a_{12}+b_{12}\right)+M\left(a_{21}+b_{21}\right)+M\left(a_{22}+b_{22}\right) \\
= & M\left(a_{11}\right)+M\left(b_{11}\right)+M\left(a_{12}\right)+M\left(b_{12}\right)+M\left(a_{21}\right)+M\left(b_{21}\right) \\
& +M\left(a_{22}\right)+M\left(b_{22}\right) \\
= & M\left(a_{11}+a_{12}+a_{21}+a_{22}\right)+M\left(b_{11}+b_{12}+b_{21}+b_{22}\right) \\
= & M(a)+M(b) .
\end{aligned}
$$

We now turn to prove that $M^{*}$ is additive. For any $x, y \in R$, there exist $a=a_{11}+a_{12}+a_{21}+a_{22}$ and $b=b_{11}+b_{12}+b_{21}+b_{22}$ in $\mathcal{G}$ such that $c=M^{*}(x+y)$ and $d=M^{*}(x)+M^{*}(y)$.

For arbitrary $s, t \in \mathcal{G}$, by the additivity of $M$, we compute

$$
\begin{aligned}
M(s a t) & =M\left(s M^{*}(x+y) t\right)=M(s)(x+y) M(t) \\
& =M(s) x M(t)+M(s) y M(t) \\
& =M\left(s M^{*}(x) t\right)+M\left(s M^{*}(y) t\right) \\
& =M\left(s M^{*}(x) t+s M^{*}(y) t\right) \\
& =M\left(s\left(M^{*}(x)+M^{*}(y)\right) t\right)=M(s b t),
\end{aligned}
$$

which implies that sat $=s b t$. Consequently, we get $a=b$, i.e., $M^{*}(x+y)=M^{*}(x)+M^{*}(y)$. 


\section{References}

[1] M. Bresar, L. Molnár and P. Serml, Elementary operators, Acta Universitatis Szegediensis, Acta Scientiarum Mathematicarum 66 (2000), 769-791.

[2] M. Bresar and P. Serml, Elementary operators, Proceedings of the Royal Society of Edinburgh: Section A Mathematics 129 (1999), 1115-1135.

[3] P. Li and F. Lu, Additivity of elementary maps on rings, Communications in Algebra 32 (2004), 3725-3737.

[4] L. Molnar and P. Serml, Elementary operators on standard operators, Linear and Multilinear Algebra 50 (2002), 315-319.

[5] Sang Og Kim and Choonkil Park, Additivity of Jordan triple product homomorphism from generalized matrix algebras, Bull. Korean Math. Soc. 50(6) (2013), 2027-2034.

[6] Y. -B. Li and Zhankui Xiao, Additivity of maps on generalized matrix algebras, A Publication of the International Linear Algebra Society 22 (2011), 743-757. 